\title{
A Hamiltonian System Method for Three-Dimensional Viscoelastic Solids
}

\author{
W.X. Zhang* and Y. Bai
}

School of Civil Engineering and Architecture, Henan University of Technology, Zhengzhou, Henan, P.R. China

\begin{abstract}
The Hamiltonian system in quasi-static problems of 3D viscoelastic solids has been introduced in this paper. Based on the principle of elastic-viscoelastic correspondence, the problem of solving partial differential equations is reduced to finding general eigensolutions of the dual equations and all the analytical fundamental eigensolutions and their corresponding Jordan forms are derived. After the establishment of symplectic adjoint relation, the final solution is expressed by linear combinations of the general eigensolutions, and the combinations are determined by the given boundary conditions. For its applications, problems of various boundary conditions and the inhomogeneous governing equations are discussed.
\end{abstract}

Keywords: Eigensolution, Quasi-static, Viscoelastic, Hamiltonian system, Boundary Conditions, Eigensolutions.

\section{INTRODUCTION}

Recently, substantive computational techniques have been found in the literature to solve engineering problems associated with viscoelastic solids and structures [1]. In these kinds of research studies, the Laplace transform method is often used as the problems of time domain can be successfully transformed into those of Laplace domain [2], and thus further discussion can be carried out in the Laplace domain. Unfortunately, researchers have found that the inverse transformation is usually very difficult to deal with. In fact, for many cases, solutions of inverse transformation cannot be expressed in an analytical way, and thus numerical inverse transformation method has been developed as an important research subject $[3,4]$. On the other hand, the timedependent stress-strain relations of viscoelasticity are often expressed by partial differential equations. This leads to the fact that analytical solutions are very difficult to be found. Therefore, numerical approaches are often considered. Among these techniques, the finite element method and the boundary element method are the most popular ones $[5,6]$.

In mechanics of elastic solids, Zhong [7] proposed the famous Hamiltonian system method. As dual variables including displacement components and stress components are used as fundamental variables, the problem of high order of partial differential equations is successfully reduced to that of lower ordinary differential ones. Moreover, the variable separation method is applied to solve the governing equations. Therefore, the original problem is further transformed into eigenvalue and eigensolution problems, and accordingly, all general solutions are obtained in the series.

However, as for viscoelastic problems, the Hamiltonian system method cannot be directly used because of the existence of the energy dissipation. It is to be noticed that by using the Laplace transform, the stress-stain relations of visco

*Address correspondence to this author at the School of Civil Engineering and Architecture, Henan University of Technology, Zhengzhou, Henan, P.R. China; Tel: 18623717351; E-mail: weixiang_zh@163.com elasticity are the same as those of elasticity in mathematics form. Xu et al. [8] analyzed two-dimensional problems of viscoelastic solids with the use of Laplace transform and the Hamiltonian formulation, and discussed a numerical method to deal with problems of displacement or stress boundary conditions and non-homogeneous governing equations.

In the present research, we studied the time-dependent property of viscoelastic solids and characteristic of the Hamiltonian formulation, and then noticed that the Hamiltonian system can also be applied to three-dimensional viscoelastic solids in the Laplace domain. We found that general solutions of the governing equation involve two parts: zero eigensolutions and non-zero eigensolutions. Zero eigensolutions are so-called Saint-Venant solutions for traditional tensional, torsional and bending problems. Non-zero eigensolutions are local effect solutions which play an important role in stress concentration problems as local effects near the boundary cannot be neglected for this case. In the numerical example, a typical boundary condition problem is discussed using the Hamiltonian system method, and the final solution is obtained by a certain linear combination of eigensolution series. The results show the creep property of viscoelastic materials when external forces are loaded on the boundary. Besides, the example well demonstrates that the Hamiltonian system provides an efficient numerical method for viscoelastic materials and structures, and is very suitable for engineering problems related with the complex boundary conditions.

\section{FUNDAMENTAL PROBLEM}

The coordinate system $(r, \theta, z)$ is selected to study a circular cylinder. The z-axis is along the longitude direction, and the origin is located at the central point of the bottom. We suppose that the behavior of the material is governed by the standard viscoelastic model shown in Fig. (1), which consists of a spring and a Kelvin model successively. Generally, the spring and Kelvin type represent the responses of instantaneous elasticity and the viscid delay, respectively. To 
simplify the theoretical derivation, the bulk behavior is assumed to be time independent in this research.

\section{I}

Fig. (1). The standard viscoelastic model.

The stress-strain relation of this viscoelastic material can be written as:

$$
\begin{gathered}
\frac{\left(G_{1}+G_{2}\right)}{G_{1}} s_{i j}(\mathbf{r}, t)+\frac{\eta}{G_{1}} \frac{\partial s_{i j}(\mathbf{r}, t)}{\partial t}=2 G_{2} e_{i j}(\mathbf{r}, \tau)+2 \eta \frac{\partial e_{i j}(\mathbf{r}, t)}{\partial t} \\
\sigma_{m m}(\mathbf{r}, t)=3 K \varepsilon_{m m}(\mathbf{r}, t)
\end{gathered}
$$

Where, $\mathbf{r}$ is the position vector, $t$ is time, $\sigma_{m m}$ and $\varepsilon_{m m}$ are the volumetric stress and strain, and $s_{i j}, e_{i j}$ are the deviatoric component tensors. According to the correspondence principle, the stress-strain relations (1) can be expressed by the following algebraic form:

$$
\begin{aligned}
& \bar{s}_{i j}(\mathbf{r}, s)=2 G^{*}(s) \bar{e}_{i j}(\mathbf{r}, s) \\
& \bar{\sigma}_{m m}(\mathbf{r}, s)=3 K \varepsilon_{m m} \bar{e}_{i j}(\mathbf{r}, s)
\end{aligned}
$$

in which, $\mathrm{s}$ is the Laplace integral parameter, and a variable with an over bar represents its counterpart in the Laplace domain. The relaxation shear modulus is:

$$
G^{*}(s)=\frac{G_{1}\left(G_{2}+\eta s\right)}{\bar{G}_{1}+\bar{G}_{2}+\eta s}
$$

The Lagrange function is:

$$
\begin{aligned}
\bar{L} & =\frac{r}{2}\left\{\lambda^{*}\left[\frac{\partial \bar{u}}{\partial r}+\frac{1}{r}\left(\bar{u}+\frac{\partial \bar{v}}{\partial \theta}\right)+\dot{\bar{w}}\right]^{2}+2 G^{*}\left[\left(\frac{\partial \bar{u}}{\partial r}\right)^{2}+\left(\frac{\bar{u}}{r}+\frac{1}{r} \frac{\partial \bar{v}}{\partial \theta}\right)^{2}+\dot{\bar{w}}^{2}\right]\right. \\
& \left.+G^{*}\left[\left(\frac{1}{r} \frac{\partial \bar{u}}{\partial \theta}+\frac{\partial \bar{v}}{\partial r}-\frac{\bar{v}}{r}\right)^{2}+\left(\dot{\bar{v}}+\frac{1}{r} \frac{\partial \bar{w}}{\partial \theta}\right)^{2}+\left(\dot{\bar{u}}+\frac{\partial \bar{w}}{\partial \theta}\right)^{2}\right]\right\}
\end{aligned}
$$

here $\lambda^{*}=K-2 G^{*} / 3$, and a variable with an over dot represents differentiation with respect to $z$. To convert the description to Hamiltonian system, the displacement components are described in vector form

$$
\overline{\mathbf{q}}=[\bar{u}, \bar{v}, \bar{w}]^{\mathrm{T}}
$$

Its dual vector

$$
\overline{\mathbf{p}}=\frac{\partial L}{\partial \dot{\overline{\mathbf{q}}}}=\left[\begin{array}{c}
r G^{*}\left(\dot{\bar{u}}+\frac{\partial \bar{w}}{\partial r}\right) \\
G^{*}\left(\frac{\partial \bar{w}}{\partial \theta}+r \dot{\bar{v}}\right) \\
\lambda^{*}\left(r \frac{\partial \bar{u}}{\partial r}+\bar{u}+\frac{\partial \bar{v}}{\partial \theta}+\dot{\bar{w}}\right)+2 G^{*} \dot{\bar{w}}
\end{array}\right]=\left[\begin{array}{c}
r \bar{\tau}_{r z} \\
r \bar{\tau}_{\theta z} \\
r \bar{\sigma}_{z}
\end{array}\right]
$$

$\delta \iiint_{\Omega} \bar{L} d r d \theta d z=0$

dual equations are obtained under the Hamiltonian system

$\dot{\bar{\psi}}=\mathbf{H} \bar{\psi}$

in which

$\bar{\psi}=\left[\begin{array}{l}\overline{\mathbf{q}} \\ \overline{\mathbf{p}}\end{array}\right]$

and the differential operator $\mathbf{H}$ is

$\left[\begin{array}{cccccc}0 & 0 & -\frac{\partial}{\partial r} & \frac{a_{3}}{r} & 0 & 0 \\ 0 & 0 & -\frac{\partial}{\partial \theta} & 0 & \frac{a_{3}}{r} & 0 \\ -a_{1} \Delta & -a_{1} \frac{\partial}{r \partial \theta} & 0 & 0 & 0 & \frac{a_{2}}{r} \\ a_{6} \frac{\partial}{r \partial \theta}-a_{5} \frac{\partial^{2}}{\partial r \partial \theta} & a_{4} \Delta^{2}-G^{*} \frac{\partial^{2}}{r \partial \theta^{2}} & 0 & 0 & 0 & a_{1} \Delta \\ -a_{5} \frac{\partial^{2}}{\partial r \partial \theta}-a_{6} \frac{\partial}{r \partial \theta} & G^{*} \Delta^{2}-a_{4} \frac{\partial^{2}}{r \partial \theta^{2}} & 0 & 0 & 0 & a_{1} \frac{\partial}{r \partial \theta} \\ 0 & 0 & 0 & \frac{\partial}{\partial r} & \frac{\partial}{\partial \theta} & 0\end{array}\right]$

here $\Delta=\partial_{r}+1 / r, \quad \Delta^{2}=1 / r-r \partial_{r^{2}}^{2}-\partial_{r}, \quad a_{1}=\lambda^{*} /\left(\lambda^{*}+2 G^{*}\right)$, $a_{2}=1 /\left(\lambda^{*}+2 G^{*}\right), \quad a_{3}=1 / G^{*}, \quad a_{4}=4 G^{*}\left(\lambda^{*}+G^{*}\right) /\left(\lambda^{*}+2 G^{*}\right)$, $a_{5}=G^{*}+2 \lambda^{*} G^{*} /\left(\lambda^{*}+2 G^{*}\right)$ and $a_{6}=G^{*}+a_{4}$.

\section{GENERAL SOLUTIONS}

Suppose the lateral boundary is free of stress; then the solution can be described as

$\bar{\psi}=\bar{\psi}_{j}(r, \theta) e^{\mu_{j} z}$

Using the variable separation method, we write the solution as

$\mathbf{H} \bar{\psi}_{j}(r, \theta)=\mu_{j} \bar{\psi}_{j}(r, \theta)$

in which, $\mu_{j}$ and $\bar{\psi}_{j}$ are eigenevalues and eigensolutions respectively. In the case of $\mu=0$, the governing equation (12) is degenerated into

$\mathbf{H} \bar{\psi}=0$

One can easily find the following fundamental solutions:

$$
\begin{aligned}
& \bar{\psi}_{1}^{(0)}=\left[\begin{array}{lllll}
\sin \theta, & \cos \theta, & 0, & 0, & 0,
\end{array}\right]^{\mathrm{T}} \\
& \bar{\psi}_{2}^{(0)}=\left[\begin{array}{llllll}
-\cos \theta, & \sin \theta, & 0, & 0, & 0, & 0
\end{array}\right]^{\mathrm{T}} \\
& \bar{\psi}_{3}^{(0)}=\left[\begin{array}{llllll}
0, & 0, & 1, & 0, & 0, & 0
\end{array}\right]^{\mathrm{T}} \\
& \bar{\psi}_{4}^{(0)}=\left[\begin{array}{llllll}
0, & r, & 0, & 0, & 0, & 0
\end{array}\right]^{\mathrm{T}}
\end{aligned}
$$

Applying the minimum potential energy principle 
Besides the fundamental eigensolutions, there exist different orders of Jordan form solutions. They are governed by

$\mathbf{H} \bar{\psi}_{i}^{(n)}=\bar{\psi}_{i}^{(n-1)} \quad(i=1,2, \cdots)$

Considering Eq. (14), we get the first order Jordan form solutions:

$\bar{\psi}_{1}^{(1)}=\left[\begin{array}{llllll}0, & 0, & -r \sin \theta, & 0, & 0, & 0\end{array}\right]^{\mathrm{T}}$

$\bar{\psi}_{2}^{(1)}=\left[\begin{array}{llllll}0, & 0, & r \cos \theta, & 0, & 0, & 0\end{array}\right]^{\mathrm{T}}$

$\bar{\psi}_{3}^{(1)}=\left[\begin{array}{llllll}-v^{*} r, & 0, & 0, & 0, & 0, & E^{*} r\end{array}\right]^{\mathrm{T}}$

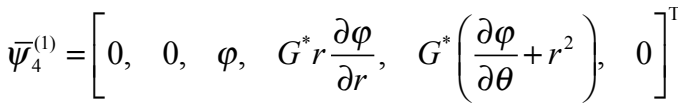

here $v^{*}=\lambda^{*} /\left[2\left(\lambda^{*}+G^{*}\right)\right], E^{*}=2 G^{*} / 2\left(1+v^{*}\right)$, and the function $\varphi$ satisfies the Neumann problem

$\nabla^{2} \varphi=\left(\frac{\partial^{2}}{\partial r^{2}}+\frac{\partial}{\partial r}+\frac{\partial^{2}}{\partial \theta^{2}}\right) \varphi=0$
$\left.\frac{d \varphi}{d n}\right|_{\partial \Omega}=-l r \sin \theta+m r \cos \theta$

For Jordan form eigensolutions, their corresponding final solutions are:

$\bar{\eta}_{i}^{(n)}=\bar{\psi}_{i}^{(n)}+z \bar{\psi}_{i}^{(n-1)}+\cdots+\frac{z^{n}}{n !} \bar{\psi}_{i}^{(0)} \quad(n=1,2, \cdots)$

Two solutions of the second Jordan form can satisfy the Jordan form equation:

$\mathbf{H} \bar{\psi}_{k}^{(2)}=\bar{\psi}_{k}^{(1)}$

They are

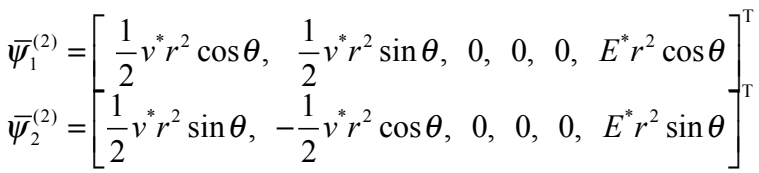

The third order Jordan form solutions are:

$$
\begin{aligned}
& \bar{\psi}_{1}^{(3)}=\left[0,0, \frac{1}{4}\left(\bar{\varphi}_{1}+r^{2} \cos \theta\right), G^{*} r \frac{\partial \bar{\varphi}_{1}}{\partial r}+\frac{1}{4}\left(3+2 v^{*}\right) G^{*} r^{3} \cos \theta,\right. \\
& \left.G^{*} \frac{\partial \bar{\varphi}_{1}}{\partial \theta}-\frac{1}{4}\left(1-2 v^{*}\right) G^{*} r^{3} \sin \theta, 0\right]^{\mathrm{T}} \\
& \bar{\psi}_{2}^{(3)}=\left[0,0, \frac{1}{4}\left(\bar{\varphi}_{2}+r^{2} \sin \theta\right), G^{*} r \frac{\partial \bar{\varphi}_{2}}{\partial r}+\frac{1}{4}\left(3+2 v^{*}\right) G^{*} r^{3} \sin \theta,\right. \\
& \left.G^{*} \frac{\partial \bar{\varphi}_{2}}{\partial \theta}+\frac{1}{4}\left(1-2 v^{*}\right) G^{*} r^{3} \cos \theta, 0\right]^{\mathrm{T}}
\end{aligned}
$$

in which the functions $\bar{\varphi}_{i}(i=1,2)$ are the solutions of the Neumann problem

$\nabla^{2} \bar{\varphi}_{j}=0$

$$
\begin{aligned}
& \left.\frac{d \bar{\varphi}_{1}}{d n}\right|_{\partial \Omega}=-\frac{1}{4}\left(3+2 v^{*}\right) r^{2} l \cos \theta+\frac{1}{4}\left(1-2 v^{*}\right) r^{2} m \sin \theta \\
& \left.\frac{d \bar{\varphi}_{2}}{d n}\right|_{\partial \Omega}=-\frac{1}{4}\left(3+2 v^{*}\right) r^{2} l \sin \theta-\frac{1}{4}\left(1-2 v^{*}\right) r^{2} m \cos \theta
\end{aligned}
$$

It can be easily proven that no other solutions can satisfy Eq. (15), and thus all zero eigensolutions are achieved. Based on the property of the Hamiltonian operator matrix $\mathrm{H}$, the integral product between the eigensolutions is defined as

$<\bar{\eta}_{1}, \mathbf{J}, \bar{\eta}_{2}>=\iint_{\Omega} \bar{\eta}_{1}^{T} \mathbf{J} \bar{\eta}_{2} d r d \theta$

Where $\bar{\eta}_{1}$ and $\bar{\eta}_{2}$ are two zero eigensolutions, and

$$
\mathbf{J}=\left[\begin{array}{cccccc}
1 & 0 & 0 & 0 & 0 & 0 \\
0 & 1 & 0 & 0 & 0 & 0 \\
0 & 0 & 1 & 0 & 0 & 0 \\
0 & 0 & 0 & -1 & 0 & 0 \\
0 & 0 & 0 & 0 & -1 & 0 \\
0 & 0 & 0 & 0 & 0 & -1
\end{array}\right]
$$

For the convenience of discussion, zero eigensolutions above are rewritten as

$$
\begin{array}{ll}
\bar{\eta}_{1}^{(\alpha)}=\bar{\eta}_{1}^{(0)}, & \bar{\eta}_{1}^{(\beta)}=\bar{\eta}_{1}^{(3)} / A_{1} \\
\bar{\eta}_{2}^{(\alpha)}=\bar{\eta}_{2}^{(0)}, & \bar{\eta}_{2}^{(\beta)}=\bar{\eta}_{2}^{(3)} / A_{2} \\
\bar{\eta}_{3}^{(\alpha)}=\bar{\eta}_{3}^{(0)}, & \bar{\eta}_{3}^{(\beta)}=\bar{\eta}_{3}^{(1)} / A_{3} \\
\bar{\eta}_{4}^{(\alpha)}=\bar{\eta}_{4}^{(0)}, & \bar{\eta}_{4}^{(\beta)}=\bar{\eta}_{4}^{(1)} / A_{4} \\
\bar{\eta}_{5}^{(\alpha)}=\bar{\eta}_{2}^{(1)}, & \bar{\eta}_{5}^{(\beta)}=\bar{\eta}_{1}^{(2)} / A_{5} \\
\bar{\eta}_{6}^{(\alpha)}=\bar{\eta}_{1}^{(1)}, & \bar{\eta}_{6}^{(\beta)}=\bar{\eta}_{2}^{(2)} / A_{6}
\end{array}
$$

Where, $\quad A_{1}=<\bar{\eta}_{1}^{(0)}, \mathbf{J}, \bar{\eta}_{1}^{(3)}>, \quad A_{2}=<\bar{\eta}_{2}^{(0)}, \mathbf{J}, \bar{\eta}_{2}^{(3)}>$, $A_{3}=<\bar{\eta}_{3}^{(0)}, \mathbf{J}, \bar{\eta}_{3}^{(1)}>, \quad A_{4}=<\bar{\eta}_{4}^{(0)}, \mathbf{J}, \bar{\eta}_{4}^{(1)}>, \quad A_{5}=<\bar{\eta}_{2}^{(1)}, \mathbf{J}, \bar{\eta}_{1}^{(2)}>$ and $A_{6}=<\bar{\eta}_{1}^{(1)}, \mathbf{J}, \bar{\eta}_{2}^{(2)}>$. Thus zero eigensolutions are classified into two groups, which satisfy the following adjoint symplectic ortho-normalization relations:

$$
\begin{aligned}
& <\bar{\eta}_{i}^{(\alpha)}, \mathbf{J}, \bar{\eta}_{j}^{(\beta)}>=-<\bar{\eta}_{i}^{(\beta)}, \mathbf{J}, \bar{\eta}_{j}^{(\alpha)}>\delta_{i j} \\
& <\bar{\eta}_{i}^{(\alpha)}, \mathbf{J}, \bar{\eta}_{j}^{(\alpha)}>=<\bar{\eta}_{i}^{(\beta)}, \mathbf{J}, \bar{\eta}_{j}^{(\beta)}>=\mathbf{0}
\end{aligned}
$$

An arbitrary vector can always be expanded by the combination of the eigensolutions

$\bar{\eta}_{c}=\sum_{n=1}^{6}\left[\bar{a}_{n} \bar{\eta}_{n}^{(\alpha)}+\bar{b}_{n} \bar{\eta}_{n}^{(\beta)}\right]$

Where

$$
\begin{aligned}
& \bar{a}_{n}=<\bar{\psi}_{c}, \mathbf{J}, \bar{\eta}_{n}^{(\beta)}> \\
& \bar{b}_{n}=-<\bar{\psi}_{c}, \mathbf{J}, \bar{\eta}_{n}^{(\alpha)}>
\end{aligned}
$$

\section{BOUNDARY CONDITIONS}

Simply by applying a variable substitution method to the governing equations (8), the inhomogeneous lateral condition can be transformed into a homogeneous one. To achieve this goal, we introduce

$$
\begin{aligned}
\bar{\psi}^{*} & =\left[\bar{u}^{*}, \bar{v}^{*}, \bar{w}^{*}, \bar{p}_{1}^{*}, \bar{p}_{2}^{*}, \bar{p}_{3}^{*}\right]^{\mathrm{T}} \\
& =\bar{\psi}-\bar{\psi}^{* *}
\end{aligned}
$$


Where

$\bar{\psi}^{* *}=\left[\bar{u}^{* *}, \bar{v}^{* *}, \bar{w}^{* *}, \bar{p}_{1}^{* *}, \bar{p}_{2}^{* *}, \bar{p}_{3}^{* *}\right]^{\mathrm{T}}$

Supposing $\bar{\psi}^{* *}$ satisfies the inhomogeneous conditions, the new variables $\bar{\psi}^{*}$ must satisfy the homogeneous lateral boundary conditions. Taking the circle cylinder for example, the inhomogeneous boundary conditions in the Hamiltonian system are:

$$
\left\{\begin{array}{l}
a_{4} \frac{\partial \bar{u}}{\partial r}+2 a_{1} G^{*}\left(\frac{\partial \bar{v}}{\partial \theta}+\bar{u}\right)+a_{1} \bar{p}_{3}=\bar{f}_{r}^{0}(\theta, z) \\
\bar{p}_{1}=\bar{f}_{z}^{0}(\theta, z) \\
G^{*}\left(\frac{\partial \bar{v}}{\partial r}+\frac{\partial \bar{u}}{\partial \theta}-\bar{v}\right)=\bar{f}_{\theta}^{0}(\theta, z)
\end{array} \quad(r=1)\right.
$$

Let

$$
\bar{\psi}^{* *}=\left[0,-a_{3} \bar{f}_{\theta}^{0}, 0, r \bar{f}_{z}^{0}, 0, r \bar{f}_{r}^{0} / a_{1}+2 r \frac{\partial \bar{f}_{\theta}^{0}}{\partial \theta}\right]^{\mathrm{T}}
$$

and

$\bar{\psi}^{*}=\left[\bar{u}, \bar{v}+a_{3} \bar{f}_{\theta}^{0}, \bar{w}, \bar{p}_{1}-\bar{f}_{z}^{0}, \bar{p}_{2}, \bar{p}_{3}-\frac{\bar{f}_{r}^{0}}{a_{1}}-2 \frac{\partial \bar{f}_{\theta}^{0}}{\partial \theta}\right]^{\mathrm{T}}$

Then the lateral boundary conditions become homogeneous, which are

$$
\left\{\begin{array}{l}
a_{4} \frac{\partial \bar{u}^{*}}{\partial r}+2 a_{1} G^{*}\left(\frac{\partial \bar{v}^{*}}{\partial \theta}+\bar{u}^{*}\right)+a_{1} \bar{p}_{3}^{*}=0 \\
\bar{p}_{1}^{*}=0 \\
\frac{\partial \bar{v}^{*}}{\partial r}+\frac{\partial \bar{u}^{*}}{\partial \theta}-\bar{v}^{*}=0
\end{array} \quad(r=1)\right.
$$

However, dual Eq. (12) should be rewritten as

$$
\dot{\bar{\psi}}^{*}=\mathbf{H} \bar{\psi}^{*}+\overline{\mathbf{f}}
$$

Where the non-homogeneous term

$$
\overline{\mathbf{f}}=\left[\begin{array}{c}
\frac{1}{r} a_{3} \bar{f}_{z}^{0} \\
a_{3} \dot{\bar{f}}_{\theta}^{0} \\
\frac{1}{r}\left[\left(a_{1} a_{3}+2 a_{2}\right) \frac{\partial \bar{f}_{\theta}^{0}}{\partial \theta}+a_{9} \bar{f}_{r}^{0}\right] \\
\frac{1}{r}\left[\bar{f}_{r}^{0}+\left(2 a_{1}-a_{3} a_{6}\right) \frac{\partial \bar{f}_{\theta}^{0}}{\partial \theta}\right]-\dot{\bar{f}}_{z}^{0} \\
\frac{1}{r}\left[-\bar{f}_{\theta}^{0}+\frac{\partial \bar{f}_{r}^{0}}{\partial \theta}+\left(a_{3} a_{4}+2 a_{1}\right) \frac{\partial^{2} \bar{f}_{\theta}^{0}}{\partial \theta^{2}}\right] \\
-a_{10}{\dot{\overline{f_{r}}}}^{0}-2 \frac{\partial \dot{\bar{f}}_{\theta}^{0}}{\partial \theta}
\end{array}\right]
$$

here $a_{9}=a_{2} / a_{1}$ and $a_{10}=1 / a_{1}$. Thus the inhomogeneous lateral boundary condition is transformed into the problem of finding a particular solution of the inhomogeneous equation. Considering the adjoint relationships of the symplectic or- thogonality, the inhomogeneous term is developed in the time domain:

$\overline{\mathbf{f}}=\sum_{n=1}^{6}\left(\bar{a}_{n} \bar{\eta}_{n}^{(\alpha)}+\bar{b}_{n} \bar{\eta}_{n}^{(\beta)}\right)$

the special solution is also described by

$\bar{\eta}_{p}=\sum_{n=1}^{6}\left[\bar{A}_{n} \bar{\eta}_{n}^{(\alpha)}+\bar{B}_{n} \bar{\eta}_{n}^{(\beta)}\right]$

where the coefficients are to be determined. Considering the governing equations (35), we have

$\dot{\bar{A}}_{n}=\dot{\bar{a}}_{n}, \dot{\bar{B}}_{n}=\dot{\bar{b}}_{n}$

The solutions are

$\bar{A}_{n}=\int_{0}^{z} \bar{a}_{n}(\xi) d \xi ; \quad \bar{B}_{n}=\int_{0}^{z} \bar{b}_{n}(\xi) d \xi$.

\section{NUMERICAL RESULTS AND DISCUSSION}

As an example, we suppose a circle cylinder under lateral boundary conditions:

$\sigma_{r}=-\sin \theta \quad(r=1)$

There are no external forces loaded at both the ends. In the numerical calculation, the Poisson's ratio is taken to be 0.25 .

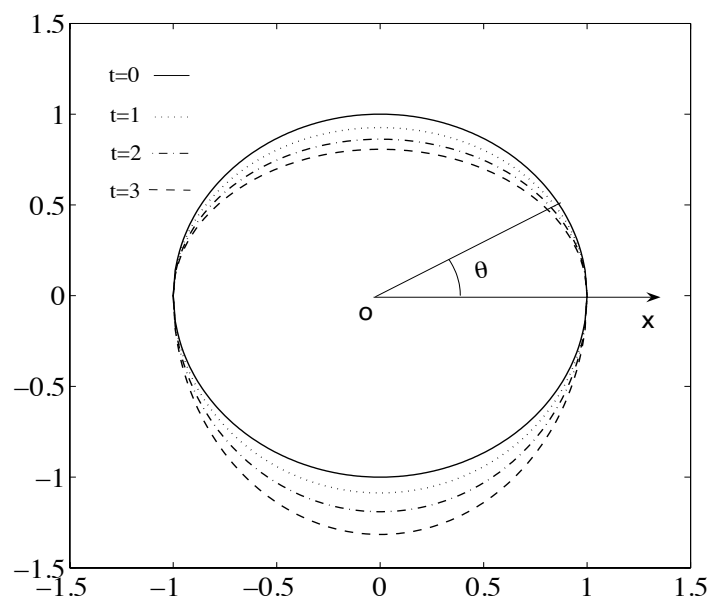

Fig. (2). The deformation of the free end.

It is clear that the example belongs to a kind of bending problem. Using the proposed method of this research, the final solution can be easily obtained. According to the numerical results, the deformation along radial direction is given in Fig. (2). It can be seen from the figure that the deformation at the free end increases with time dramatically, which explains that viscoelastic materials have the property of displacement creep. Besides, at time $t=0$, certain initial deformation can be found from the figure. This phenomenon suggests that the material has some initial response to external loads.

\section{CONCLUSION}

Considering the principle of elastic-viscoelastic correspondence and the time-dependent character of viscoelastic 
solids, we studied three dimensional viscoelastic problems under the Hamiltonian system. The variable separation method was applied to reduce the high order partial differential governing equations, and therefore the final solution can be described by series of eigensolutions. In fact, all solutions of traditional Saint-Venant problems including tension, torsion and bending deformations can be obtained by combinations of the eigensolutions. When the inhomogeneous boundary conditions are considered, the variable substitution method is applied, and accordingly the problem is successfully transformed into solving inhomogeneous governing equations. According to the adjoint symplectic orthogonality relations, the special solutions can be achieved analytically by using the technology of the eigensolution expansion.

\section{CONFLICT OF INTEREST}

The authors confirm that this article content has no conflict of interest.

\section{ACKNOWLEDGEMENTS}

Declared none.

\section{REFERENCES}

[1] L.Q. Chen, H. Chen and C.W. Lim, "Asymptotic analysis of axially accelerating viscoelastic strings", International Journal of Engineering Science, vol. 46, no. 10, pp. 976-985, 2008.

[2] J. Brilla, "Laplace transform and new mathematical theory of viscoelasticity", Meccanica, vol. 32, pp. 187-195, 1997.

[3] L.J. De Chant, "Impulsive displacement of a quasi-linear viscoelastic material through accurate numerical inversion of the laplace transform", Computers \& Mathematics with Applications, vol. 43, pp. 1161-1170, 2002.

[4] M.A. Ezzat, A.S. El-Karamany, A.A. Samaan, and M. Zakaria, "The relaxation effects of the volume properties of viscoelastic material in generalized thermoelasticity with thermal relaxation", Journal of Thermal Stresses, vol. 26, pp. 671-690, 2003.

[5] M. Schanz, H. Antes and T. Rüberg, "Convolution quadrature boundary element method for quasi-static visco- and poroelastic continua", Computers \& Structures, vol. 83, pp. 673-684, 2005.

[6] A.D. Mesquita and H.B. Coda, "A boundary element methodology for viscoelastic analysis: Part I with cells", Applied Mathematical Modelling, vol. 31, pp. 1149-1170, 2007.

[7] W.X. Zhong, "On the reciprocal theorem and adjoint symplectic orthogonal relation", Acta Mechanica Sinica, vol. 24, pp. 432-437, 1992

[8] X.S. Xu, W.X. Zhang, X. Li and G.P. Wang, "An application of the symplectic system in two-dimensional viscoelasticity", International Journal of Engineering Science, vol. 44, pp. 897-914, 2006.

Received: June 10,2015

(C) Zhang and Bai; Licensee Bentham Open.

This is an open access article licensed under the terms of the (https://creativecommons.org/licenses/by/4.0/legalcode), which permits unrestricted, noncommercial use, distribution and reproduction in any medium, provided the work is properly cited. 